\title{
On the Solvability of Caputo $q$-Fractional Boundary Value Problem Involving $p$-Laplacian Operator
}

\author{
Hüseyin Aktuğlu and Mehmet Ali Özarslan \\ Eastern Mediterranean University, Gazimagusa, Mersin 10, Turkey \\ Correspondence should be addressed to Hüseyin Aktuğlu; huseyin.aktuglu@emu.edu.tr
}

Received 2 April 2013; Accepted 10 June 2013

Academic Editor: Nazim Idrisoglu Mahmudov

Copyright () 2013 H. Aktuğlu and M. Ali Özarslan. This is an open access article distributed under the Creative Commons Attribution License, which permits unrestricted use, distribution, and reproduction in any medium, provided the original work is properly cited.

We consider the model of a Caputo $q$-fractional boundary value problem involving $p$-Laplacian operator. By using the Banach contraction mapping principle, we prove that, under some conditions, the suggested model of the Caputo $q$-fractional boundary value problem involving $p$-Laplacian operator has a unique solution for both cases of $0<p<1$ and $p>2$. It is interesting that in both cases solvability conditions obtained here depend on $q, p$, and the order of the Caputo $q$-fractional differential equation. Finally, we illustrate our results with some examples.

\section{Introduction}

In this section we will give some basic definitions and results that will be needed in the sequel. For more details about the theory of $q$-calculus, fractional calculus, and $q$-fractional calculus, we refer readers to [1-10].

Let $q \in(0,1)$ be a fixed real number. Then for any $\alpha \in \mathbb{R}$,

$$
[\alpha]_{q}:=\frac{1-q^{\alpha}}{1-q} .
$$

The $q$-binomial function is defined for all $n \in \mathbb{N}$ as

$$
\begin{gathered}
(t-s)_{q}^{n}=\prod_{k=0}^{n-1}\left(t-q^{k} s\right), \\
(t-s)_{q}^{\beta}=t^{\beta} \prod_{i=0}^{\infty}\left(\frac{1-(s / t) q^{i}}{1-(s / t) q^{i+\alpha}}\right),
\end{gathered}
$$

where $\beta$ is not a positive integer. It is easy to see that

$$
(a t-a s)_{q}^{\beta}=a^{\beta}(t-s)_{q}^{\beta} .
$$

The $q$-analog of Euler's gamma function is denoted by $\Gamma_{q}(t)$ and defined as

$$
\Gamma_{q}(t)=\frac{(1-q)_{q}^{t-1}}{(1-q)^{t-1}}, \quad t>0 .
$$

The following theorem will be used to compare values of $\Gamma(t)$, the usual gamma function, with values of $\Gamma_{q}(t)$ for a fixed $q \epsilon$ $(0,1)$.

Theorem 1 (see [11]). For $0<r<q<1$, one has

$$
\begin{gathered}
\Gamma_{r}(t) \leq \Gamma_{q}(t) \leq \Gamma(t), \quad \text { for } 0<t \leq 1 \text { or } t \geq 2, \\
\Gamma(t) \leq \Gamma_{q}(t) \leq \Gamma_{r}(t), \quad \text { for } 1 \leq t \leq 2 .
\end{gathered}
$$

It is known that for $0<q<1$,

$$
\begin{aligned}
\mathbb{T}_{q} & =\left\{q^{n} ; n \in \mathbb{Z}\right\} \cup\{0\}, \\
\mathbb{T}_{q}^{\alpha}=\left\{q^{n+\alpha} ; n\right. & \in \mathbb{Z}\} \cup\{0\}, \quad \alpha \in \mathbb{R}^{+} \cup\{0\} .
\end{aligned}
$$

The nabla $q$-derivative of the function $f: \mathbb{T}_{q} \rightarrow \mathbb{R}$ is defined by

$$
\nabla_{q} f(s)=\frac{f(s)-f(q s)}{(1-q) s}, \quad s \in \mathbb{T}_{q}-\{0\} .
$$


The nabla $q$-integral of $f$ is defined by

$$
\int_{0}^{s} f(t) \nabla_{q} t=(1-q) s \sum_{k=0}^{\infty} q^{k} f\left(s q^{k}\right) .
$$

Jackson in [12] and Thomae in [13] showed that the q-beta function, which is defined by

$$
B_{q}(t, s)=\frac{\Gamma_{q}(t) \Gamma_{q}(s)}{\Gamma_{q}(t+s)}
$$

has the following $q$-integral representation:

$$
B_{q}(t, s)=\int_{0}^{1} \tau^{t-1}(1-q \tau)_{q}^{s-1} \nabla_{q} \tau, \quad t, s>0 .
$$

The fundamental theorem of $q$-calculus states that

$$
\nabla_{q} \int_{0}^{s} f(t) \nabla_{q} t=f(s)
$$

and if $f$ is continuous at 0 , then

$$
\int_{0}^{s} \nabla_{q} f(t) \nabla_{q} t=f(s)-f(0) .
$$

Moreover,

$$
\nabla_{q} \int_{0}^{t} f(t, s) \nabla_{q} s=\int_{0}^{t} \nabla_{q} f(t, s) \nabla_{q} s+f(q t, t),
$$

where the derivative is applied with respect to $t$.

The nabla $q$-fractional derivative of $(t-s)_{q}^{\alpha}$ with respect to $t$ and for all $\alpha \in \mathbb{R}$ is given by

$$
\nabla_{q}(t-s)_{q}^{\alpha}=\frac{1-q^{\alpha}}{1-q}(t-s)_{q}^{\alpha-1} .
$$

Moreover, the $q$-fractional integral of order $\alpha \neq 0,-1,-2, \ldots$ is defined by

$$
{ }_{q} I_{0}^{\alpha} f(t)=\frac{1}{\Gamma_{q}(\alpha)} \int_{0}^{t}(t-q s)_{q}^{\alpha-1} f(s) \nabla_{q} s .
$$

The $\alpha$-order Caputo $q$-fractional derivative of a function $f$ is defined by

$$
{ }_{q} C_{0}^{\alpha} f(t)=\frac{1}{\Gamma_{q}(n-\alpha)} \int_{0}^{t}(t-q s)^{n-\alpha-1} \nabla_{q}^{n} f(s) \nabla_{q} s,
$$

where $n=[\alpha]+1$ and $[\alpha]$ denotes the greatest integer less than or equal to $\alpha$.

The following lemma enables us to transfer Caputo $q$-fractional differential equations into an equivalent $q$-fractional integral equation.

Lemma 2 (see [3]). Assume that $\alpha>0$ and $f$ is defined on a suitable domain. Then

$$
{ }_{q} I_{0 q}^{\alpha} C_{0}^{\alpha} f(t)=f(t)-\sum_{i=0}^{n-1} \frac{t^{k}}{\Gamma_{q}(k+1)} \nabla_{q}^{k} f(0)
$$

and if $0<\alpha \leq 1$, then

$$
q_{0 q}^{I_{0 q}^{\alpha}} C_{0}^{\alpha} f(t)=f(t)-f(0) .
$$

On the other hand the operator $\varphi_{p}(s)=|s|^{p-2} s$, where $p>1$ is called the $p$-Laplacian operator. It is easy to see that $\varphi_{p}^{-1}=\varphi_{r}$, where $(1 / p)+(1 / r)=1$. The following properties of $p$-Laplacian operator will be used in the rest of the paper.

(P1) if $1<p<2, x y>0$, and $|x|,|y| \geq m>0$, then $\left|\varphi_{p}(x)-\varphi_{p}(y)\right| \leq(p-1) m^{p-2}|x-y| ;$

(P2) if $p \geq 2$ and $|x|,|y| \leq M$ then, $\left|\varphi_{p}(x)-\varphi_{p}(y)\right| \leq$ $(p-1) M^{p-2}|x-y|$.

\section{A Model of Caputo $q$-Fractional Boundary Value Problem Involving $p$-Laplacian Operator}

In this paper, our main aim is to prove the existence and uniqueness of the solution for the following Caputo $q$-fractional boundary value problem involving the $p$-Laplacian operator:

$$
\begin{gathered}
\nabla_{q}\left(\varphi_{p}\left({ }_{q} C_{0}^{\alpha} x(t)\right)\right)=f(t, x(t)), \\
\nabla_{q}^{k} x(0)=0, \quad \text { for } k=2,3, \ldots, n-1, \\
x(0)=a_{0} x(1), \\
\nabla_{q} x(0)=a_{1} \nabla_{q} x(1),
\end{gathered}
$$

where $a_{0}, a_{1} \neq 1,1<\alpha \in \mathbb{R}$, and $f \in C([0,1] \times \mathbb{R}, \mathbb{R})$.

Note that, the boundary value problem given in (19) is antiperiodic for $a_{0}, a_{1}=-1$.

In the following lemma we obtain a $q$-integral equation which is equivalent to the Caputo $q$-fractional boundary value problem given in (19).

Lemma 3. Assume that $\alpha>1, a_{0}, a_{1} \neq 1$, and $h \in C([0,1])$. Then

$$
\begin{gathered}
\nabla_{q}\left(\varphi_{p}\left({ }_{q} C_{0}^{\alpha} x(t)\right)\right)=h(t), \\
\nabla_{q}^{k} x(0)=0, \quad \text { for } k=2,3, \ldots, n-1, \\
x(0)=a_{0} x(1), \\
\nabla_{q} x(0)=a_{1} \nabla_{q} x(1)
\end{gathered}
$$

are equivalent to the following q-integral equation:

$$
\begin{aligned}
x(t)= & b_{0}^{q} \int_{0}^{t}(t-q \tau)_{q}^{\alpha-1} \varphi_{r}\left(\int_{0}^{\tau} h(s) \nabla_{q} s\right) \nabla_{q} \tau \\
& +b_{1}^{q} \int_{0}^{1}(1-q \tau)_{q}^{\alpha-1} \varphi_{r}\left(\int_{0}^{\tau} h(s) \nabla_{q} s\right) \nabla_{q} \tau \\
& +b_{2}^{q}(t) \int_{0}^{1}(1-q \tau)_{q}^{\alpha-2} \varphi_{r}\left(\int_{0}^{\tau} h(s) \nabla_{q} s\right) \nabla_{q} \tau,
\end{aligned}
$$

where $b_{0}^{q}=1 /\left(\Gamma_{q}(\alpha)\right), b_{1}^{q}=a_{0} /\left(\Gamma_{q}(\alpha)\left(1-a_{0}\right)\right)$, and $b_{2}^{q}(t)=$ $\left(a_{1}\left(t+a_{0}(1-t)\right)\right) /\left(\Gamma_{q}(\alpha-1)\left(1-a_{0}\right)\left(1-a_{1}\right)\right)$. 
Proof. Using (20) and the fact that $\varphi_{p}\left({ }_{q} C_{0}^{\alpha} x(0)\right)=0$, we have

$$
\varphi_{p}\left({ }_{q} C_{0}^{\alpha} x(t)\right)=\int_{0}^{t} h(s) \nabla_{q} s
$$

or equivalently,

$$
{ }_{q} C_{0}^{\alpha} x(t)=\varphi_{r}\left(\int_{0}^{t} h(s) \nabla_{q} s\right)
$$

Applying $q$-fractional integral operator ${ }_{q} I_{0}^{\alpha}$ to both sides and using Lemma 2, we get

$$
\begin{aligned}
x(t) & -\sum_{k=0}^{n-1} \frac{t^{k}}{\Gamma_{q}(k+1)} \nabla_{q}^{k} x(0) \\
= & \frac{1}{\Gamma_{q}(\alpha)} \int_{0}^{t}(t-q \tau)_{q}^{\alpha-1} \varphi_{r}\left(\int_{0}^{\tau} h(s) \nabla_{q} s\right) \nabla_{q} \tau .
\end{aligned}
$$

Using $\nabla_{q}^{k} x(0)=0$, for $k=2,3, \ldots,[\alpha]-1$ in $(24)$, we obtain

$$
\begin{aligned}
x(t)= & \frac{1}{\Gamma_{q}(\alpha)} \int_{0}^{t}(t-q \tau)_{q}^{\alpha-1} \varphi_{r}\left(\int_{0}^{\tau} h(s) \nabla_{q} s\right) \nabla_{q} \tau \\
& +x(0)+t \nabla_{q} x(0) .
\end{aligned}
$$

According to (13) and (14), we have

$$
\begin{aligned}
\nabla_{q} x(t)= & \frac{1}{\Gamma_{q}(\alpha-1)} \int_{0}^{t}(t-q \tau)_{q}^{\alpha-2} \varphi_{r}\left(\int_{0}^{\tau} h(s) \nabla_{q} s\right) \nabla_{q} \tau \\
& +\nabla_{q} x(0)
\end{aligned}
$$

Taking $t=1$ in both sides of (25) and (26), we get

$$
\begin{aligned}
x(1)= & \frac{1}{\Gamma_{q}(\alpha)} \int_{0}^{1}(1-q \tau)_{q}^{\alpha-1} \varphi_{r}\left(\int_{0}^{\tau} h(s) \nabla_{q} s\right) \nabla_{q} \tau \\
& +x(0)+\nabla_{q} x(0), \\
\nabla_{q} x(1)= & \frac{1}{\Gamma_{q}(\alpha-1)} \int_{0}^{1}(1-q \tau)_{q}^{\alpha-2} \varphi_{r}\left(\int_{0}^{\tau} h(s) \nabla_{q} s\right) \nabla_{q} \tau \\
& +\nabla_{q} x(0) .
\end{aligned}
$$

Solving equations obtained by the given boundary value conditions $x(0)=a_{0} x(1)$ and $\nabla_{q} x(0)=a_{1} \nabla_{q} x(1)$, it follows that

$$
\begin{aligned}
\nabla_{q} x(0)= & \frac{a_{1}}{\Gamma_{q}(\alpha-1)\left(1-a_{1}\right)} \\
& \times \int_{0}^{1}(1-q \tau)_{q}^{\alpha-2} \varphi_{r}\left(\int_{0}^{\tau} h(s) \nabla_{q} s\right) \nabla_{q} \tau \\
x(0)= & \frac{a_{0}}{\Gamma_{q}(\alpha)\left(1-a_{0}\right)} \int_{0}^{1}(1-q \tau)_{q}^{\alpha-1} \varphi_{r}\left(\int_{0}^{\tau} h(s) \nabla_{q} s\right) \nabla_{q} \tau
\end{aligned}
$$

$$
\begin{aligned}
& +\frac{a_{0}}{\Gamma_{q}(\alpha-1)\left(1-a_{0}\right)\left(1-a_{1}\right)} \\
& \times \int_{0}^{1}(1-q \tau)_{q}^{\alpha-2} \varphi_{r}\left(\int_{0}^{\tau} h(s) \nabla_{q} s\right) \nabla_{q} \tau .
\end{aligned}
$$

Substituting (28) into (25) gives (21) which completes the proof.

\section{Solvability of the Caputo $q$-Fractional Boundary Value Problem}

This section is devoted to the solvability of the Caputo $q$ fractional boundary value problem given in (19). In the first part we shall prove the existence and uniqueness of the solution, and then we shall illustrate our main results with some examples.

Recall that $C[0,1]$ is a Banach space with the norm $\|x\|=$ $\max _{t \in[0,1]}|x(t)|$. Now consider $T_{i}: C[0,1] \rightarrow C[0,1], i=$ 0,1 with

$$
\begin{gathered}
T_{0} x(t):=\varphi_{r}\left(\int_{0}^{t} f(s, x(s)) \nabla_{q} s\right) \\
T_{1} x(t)=b_{0}^{q} \int_{0}^{t}(t-q \tau)_{q}^{\alpha-1} x(\tau) \nabla_{q} \tau \\
+b_{1}^{q} \int_{0}^{1}(1-q \tau)_{q}^{\alpha-1} x(\tau) \nabla_{q} \tau \\
+b_{2}^{q}(t) \int_{0}^{1}(1-q \tau)_{q}^{\alpha-2} x(\tau) \nabla_{q} \tau .
\end{gathered}
$$

Then $T=T_{1} \circ T_{0}$ is a continuous and compact operator.

Theorem 4. Suppose that $1<r<2, a_{0}, a_{1} \neq 1, q \in(0,1)$ is fixed, and the following conditions hold: $\exists \lambda>0,0<\delta<$ $2 /(2-r)$ and $d$ with

$$
\begin{aligned}
0< & d \\
< & \lambda^{2-r} \frac{\Gamma_{q}(\delta(r-2)+2+\alpha)}{(r-1) \Gamma_{q}(\delta(r-2)+2)} \\
& \times\left[\frac{\left|1-a_{0}\right|\left|1-a_{1}\right|}{\left(\left|1-a_{0}\right|+\left|a_{0}\right|\right)\left(\left|1-a_{1}\right|+\left|a_{1}\right|[\delta(r-2)+\alpha+1]_{q}\right)}\right]
\end{aligned}
$$

such that

$$
\begin{aligned}
& {[\delta]_{q} \lambda t^{\delta-1} \leq f(t, x), \quad \text { for any }(t, x) \in(0,1] \times \mathbb{R},} \\
& |f(t, x)-f(t, y)| \leq d|x-y|, \quad \text { for } t \in[0,1], x, y \in \mathbb{R} .
\end{aligned}
$$

Then the boundary value problem (19) has a unique solution. 
Proof. Inequality given in (31) implies that

$$
\lambda t^{\delta} \leq \int_{0}^{t} f(s, x) \nabla_{q} s, \quad \text { for any }(t, x) \in[0,1] \times \mathbb{R} .
$$

On the other hand using (P1) and (32), we have

$$
\begin{aligned}
& \left|T_{0} x(t)-T_{0} y(t)\right| \\
& \quad=\left|\varphi_{r}\left(\int_{0}^{t} f(s, x(s)) \nabla_{q} s\right)-\varphi_{r}\left(\int_{0}^{t} f(s, y(s)) \nabla_{q} s\right)\right| \\
& \quad \leq(r-1)\left(\lambda t^{\delta}\right)^{r-2}\left|\int_{0}^{t} f(s, x(s)) \nabla_{q} s-\int_{0}^{t} f(s, y(s)) \nabla_{q} s\right| \\
& \quad \leq(r-1)\left(\lambda t^{\delta}\right)^{r-2} \int_{0}^{t}|f(s, x(s))-f(s, y(s))| \nabla_{q} s \\
& \quad \leq d(r-1)\left(\lambda t^{\delta}\right)^{r-2} \int_{0}^{t}|x(s)-y(s)| \nabla_{q} s \\
& \quad \leq d(r-1) \lambda^{r-2} t^{\delta(r-2)+1}\|x-y\| .
\end{aligned}
$$

Similarly,

$$
\begin{aligned}
& |T x(t)-T y(t)| \\
& =\left|T_{1}\left(T_{0} x(t)\right)-T_{1}\left(T_{0} y(t)\right)\right| \\
& =\mid b_{0}^{q} \int_{0}^{t}(t-q \tau)_{q}^{\alpha-1}\left(\left(T_{0} x\right)(\tau)-\left(T_{0} y\right)(\tau)\right) \nabla_{q} \tau \\
& \quad+b_{1}^{q} \int_{0}^{1}(1-q \tau)_{q}^{\alpha-1}\left(\left(T_{0} x\right)(\tau)-\left(T_{0} y\right)(\tau)\right) \nabla_{q} \\
& \quad+b_{2}^{q}(t) \int_{0}^{1}(1-q \tau)_{q}^{\alpha-2}\left(\left(T_{0} x\right)(\tau)-\left(T_{0} y\right)(\tau)\right) \nabla_{q} \tau \mid .
\end{aligned}
$$

Finally using (34) in (35), we get

$$
\begin{aligned}
& |T x(t)-T y(t)| \\
& \leq d(r-1) \lambda^{r-2}\|x-y\| \\
& \times\left[b_{0}^{q} \int_{0}^{t}(t-q \tau)_{q}^{\alpha-1} \tau^{\delta(r-2)+1} \nabla_{q} \tau\right. \\
& \quad+\left|b_{1}^{q}\right| \int_{0}^{1}(1-q \tau)_{q}^{\alpha-1} \tau^{\delta(r-2)+1} \nabla_{q} \tau \\
& \left.\quad+\left|b_{2}^{q}(t)\right| \int_{0}^{1}(1-q \tau)_{q}^{\alpha-2} \tau^{\delta(r-2)+1} \nabla_{q} \tau\right] .
\end{aligned}
$$

Since

$$
\begin{aligned}
& \int_{0}^{t}(t-q \tau)_{q}^{\alpha-1} \tau^{\delta(r-2)+1} \nabla_{q} \tau \\
& \quad=\int_{0}^{1}(1-q \tau)_{q}^{\alpha-1} t^{\delta(r-2)+\alpha+1} \tau^{\delta(r-2)+1} \nabla_{q} \tau
\end{aligned}
$$

we have

$$
\begin{aligned}
& |T x(t)-T y(t)| \\
& \leq d(r-1) \lambda^{r-2}\|x-y\| \\
& \times\left[b_{0}^{q} \int_{0}^{1}(1-q \tau)_{q}^{\alpha-1} t^{\delta(r-2)+\alpha+1} \tau^{\delta(r-2)+1} \nabla_{q} \tau\right. \\
& +\left|b_{1}^{q}\right| \int_{0}^{1}(1-q \tau)_{q}^{\alpha-1} \tau^{\delta(r-2)+1} \nabla_{q} \tau \\
& \left.+\left|b_{2}^{q}(t)\right| \int_{0}^{1}(1-q \tau)_{q}^{\alpha-2} \tau^{\delta(r-2)+1} \nabla_{q} \tau\right] \\
& =d(r-1) \lambda^{r-2}\|x-y\| \\
& \times\left[b_{0}^{q} t^{\delta(r-2)+\alpha+1} B_{q}(\delta(r-2)+2, \alpha)\right. \\
& +\left|b_{1}^{q}\right| B_{q}(\delta(r-2)+2, \alpha) \\
& \left.+\left|b_{2}^{q}(t)\right| B_{q}(\delta(r-2)+2, \alpha-1)\right] \\
& =d(r-1) \lambda^{r-2}\|x-y\| \\
& \times\left[b_{0}^{q} t^{\delta(r-2)+\alpha+1} B_{q}(\delta(r-2)+2, \alpha)\right. \\
& +\left|b_{1}^{q}\right| B_{q}(\delta(r-2)+2, \alpha)+\left|b_{2}^{q}(t)\right| \\
& \left.\times \frac{[\delta(r-2)+\alpha+1]_{q}}{[\alpha-1]_{q}} B_{q}(\delta(r-2)+2, \alpha)\right] \\
& \leq d(r-1) \lambda^{r-2}\|x-y\| B_{q}(\delta(r-2)+2, \alpha) \\
& \times\left[b_{0}^{q} t^{\delta(r-2)+\alpha+1}+\left|b_{1}^{q}\right|+\left|b_{2}^{q}(t)\right| \frac{[\delta(r-2)+\alpha+1]_{q}}{[\alpha-1]_{q}}\right] .
\end{aligned}
$$

In other words,

$$
\begin{aligned}
& |T x(t)-T y(t)| \\
& \leq d(r-1) \lambda^{r-2}\|x-y\| \frac{\Gamma_{q}(\delta(r-2)+2) \Gamma_{q}(\alpha)}{\Gamma_{q}(\delta(r-2)+2+\alpha)} \\
& \times \quad\left[\frac{1}{\Gamma_{q}(\alpha)} t^{\delta(r-2)+\alpha+1}+\left|\frac{a_{0}}{\Gamma_{q}(\alpha)\left(1-a_{0}\right)}\right|\right. \\
& \left.\quad+\left|\frac{a_{1}\left(t+a_{0}(1-t)\right)}{\Gamma_{q}(\alpha-1)\left(1-a_{0}\right)\left(1-a_{1}\right)}\right| \frac{[\delta(r-2)+\alpha+1]_{q}}{[\alpha-1]_{q}}\right] \\
& \leq d(r-1) \lambda^{r-2} \| x-y \mid \frac{\Gamma_{q}(\delta(r-2)+2) \Gamma_{q}(\alpha)}{\Gamma_{q}(\delta(r-2)+2+\alpha)} \\
& \times \\
& \quad\left[\frac{1}{\Gamma_{q}(\alpha)}+\frac{\left|a_{0}\right|}{\Gamma_{q}(\alpha)\left|1-a_{0}\right|}\right. \\
& \left.\quad+\frac{\left|a_{1}\right|\left(\left|a_{0}\right|+\left|1-a_{0}\right|\right)[\delta(r-2)+\alpha+1]_{q}}{\Gamma_{q}(\alpha)\left|1-a_{0}\right|\left|1-a_{1}\right|}\right]
\end{aligned}
$$




$$
\begin{aligned}
& \leq d(r-1) \lambda^{r-2}\|x-y\| \frac{\Gamma_{q}(\delta(r-2)+2) \Gamma_{q}(\alpha)}{\Gamma_{q}(\delta(r-2)+2+\alpha)} \\
& \times\left[\left(\left|1-a_{0}\right|\left|1-a_{1}\right|+\left|a_{0}\right|\left|1-a_{1}\right|\right.\right. \\
& \left.\quad+\left|a_{1}\right|\left(\left|a_{0}\right|+\left|1-a_{0}\right|\right)[\delta(r-2)+\alpha+1]_{q}\right) \\
& \left.\quad \times\left(\Gamma_{q}(\alpha)\left|1-a_{0}\right|\left|1-a_{1}\right|\right)^{-1}\right] \\
& =d(r-1) \lambda^{r-2} \frac{\Gamma_{q}(\delta(r-2)+2)}{\Gamma_{q}(\delta(r-2)+2+\alpha)} \\
& \times\left[\frac{\left(\left|1-a_{0}\right|+\left|a_{0}\right|\right)\left(\left|1-a_{1}\right|+\left|a_{1}\right|[\delta(r-2)+\alpha+1]_{q}\right)}{\left|1-a_{0}\right|\left|1-a_{1}\right|}\right] \\
& \quad \times\|x-y\|=K\|x-y\|,
\end{aligned}
$$

where $K=d(r-1) \lambda^{r-2}\left(\Gamma_{q}(\delta(r-2)+2) / \Gamma_{q}(\delta(r-2)+2+\alpha)\right)[((\mid 1-$ $\left.\left.\left.a_{0}|+| a_{0} \mid\right)\left(\left|1-a_{1}\right|+\left|a_{1}\right|[\delta(r-2)+\alpha+1]_{q}\right)\right) /\left(\left|1-a_{0}\right|\left|1-a_{1}\right|\right)\right]$.

By condition (30), we get $0<K<1$, which implies that $T$ is a contraction. As a consequence of the Banach contraction mapping theorem and Lemma 3, the boundary value problem given in (19) has a unique solution.

Theorem 5. Suppose that $1<r<2, a_{0}, a_{1} \neq 1$, and the following conditions hold for a fixed $q \in(0,1), \exists \lambda>0,0<\delta<$ $2 /(2-r)$, and $d$ with

$$
\begin{aligned}
0< & d \\
< & \lambda^{2-r} \frac{\Gamma_{q}(\delta(r-2)+2+\alpha)}{(r-1) \Gamma_{q}(\delta(r-2)+2)} \\
& \times\left[\frac{\left|1-a_{0}\right|\left|1-a_{1}\right|}{\left(\left|1-a_{0}\right|+\left|a_{0}\right|\right)\left(\left|1-a_{1}\right|+\left|a_{1}\right|[\delta(r-2)+\alpha+1]_{q}\right)}\right]
\end{aligned}
$$

such that

$$
\begin{gathered}
f(t, x) \leq-[\delta]_{q} \lambda t^{\delta-1}, \quad \text { for any }(t, x) \in(0,1] \times \mathbb{R}, \\
|f(t, x)-f(t, y)| \leq d|x-y|, \quad \text { for } t \in[0,1], x, y \in \mathbb{R} .
\end{gathered}
$$

Then the boundary value problem (19) has a unique solution.

Remark 6. When $q \rightarrow 1$, Theorems 4 and 5 reduce to Theorems 3.1 and 3.2 of [14].

Theorem 7. Suppose that $r>2, a_{0}, a_{1} \neq 1$, and the following conditions hold for a fixed $q \in(0,1)$. There exists a nonnegative function $g(x) \in L[0,1]$ with $M:=\int_{0}^{1} g(\tau) \nabla_{q} \tau \geq 0$ such that

$$
|f(t, x)| \leq g(t), \quad \text { for any }(t, x) \in[0,1] \times \mathbb{R}
$$

and there exists a constant $d$ with

$$
\begin{aligned}
& 0<d \\
& <\frac{\Gamma_{q}(\alpha+2)}{(r-1) M^{r-2}} \\
& \quad \times\left[\frac{\left|1-a_{0}\right|\left|1-a_{1}\right|}{\left(\left|1-a_{0}\right|+\left|a_{0}\right|\right)\left(\left|1-a_{1}\right|+\left|a_{1}\right|[\alpha+1]_{q}\right)}\right] \\
& |f(t, x)-f(t, y)| \leq d|x-y|, \quad \text { for } t \in[0,1], x, y \in \mathbb{R} .
\end{aligned}
$$

Then the boundary value problem (19) has a unique solution.

Proof. By (42), we can get that

$$
\int_{0}^{t}|f(\tau, x(\tau))| \nabla_{q} \tau \leq \int_{0}^{1} g(\tau) \nabla_{q} \tau=M
$$

for all $t \in[0,1]$. By the definition of $T_{0}$, we have

$$
\begin{aligned}
& \left|T_{0} x(t)-T_{0} y(t)\right| \\
& \quad=\left|\varphi_{r}\left(\int_{0}^{t} f(s, x(s)) \nabla_{q} s\right)-\varphi_{r}\left(\int_{0}^{t} f(s, y(s)) \nabla_{q} s\right)\right| .
\end{aligned}
$$

Using (P2) and (45) gives

$$
\begin{aligned}
\left|T_{0} x(t)-T_{0} y(t)\right| & \\
\leq & (r-1) M^{r-2} \\
& \times\left|\int_{0}^{t} f(s, x(s)) \nabla_{q} s-\int_{0}^{t} f(s, y(s)) \nabla_{q} s\right| \\
\leq & (r-1) M^{r-2} \\
& \times \int_{0}^{t}|f(s, x(s))-f(s, y(s))| \nabla_{q} s \\
\leq & d(r-1) M^{r-2} \int_{0}^{t}|x(s)-y(s)| \nabla_{q} s \\
\leq & d(r-1) M^{r-2} t\|x-y\| .
\end{aligned}
$$

Therefore,

$$
\begin{aligned}
& |T x(t)-T y(t)| \\
& =\left|T_{1}\left(T_{0} x(t)\right)-T_{1}\left(T_{0} y(t)\right)\right| \\
& =\mid b_{0}^{q} \int_{0}^{t}(t-q \tau)_{q}^{\alpha-1}\left(\left(T_{0} x\right)(\tau)-\left(T_{0} y\right)(\tau)\right) \nabla_{q} \tau \\
& \quad+b_{1}^{q} \int_{0}^{1}(1-q \tau)_{q}^{\alpha-1}\left(\left(T_{0} x\right)(\tau)-\left(T_{0} y\right)(\tau)\right) \nabla_{q} \\
& \quad+b_{2}^{q}(t) \int_{0}^{1}(1-q \tau)_{q}^{\alpha-2}\left(\left(T_{0} x\right)(\tau)-\left(T_{0} y\right)(\tau)\right) \nabla_{q} \tau \mid
\end{aligned}
$$




$$
\begin{aligned}
& \leq d(r-1) M^{r-2}\|x-y\| \\
& \times\left[\frac{1}{\Gamma_{q}(\alpha)} \int_{0}^{t}(t-q \tau)_{q}^{\alpha-1} \tau \nabla_{q} \tau\right. \\
& \quad+\frac{\left|a_{0}\right|}{\Gamma_{q}(\alpha)\left|1-a_{0}\right|} \int_{0}^{1}(1-q \tau)_{q}^{\alpha-1} \tau \nabla_{q} \tau \\
& \left.\quad+\frac{\left|a_{1}\left(t+a_{0}(1-t)\right)\right|}{\Gamma_{q}(\alpha-1)\left|1-a_{0}\right|\left|1-a_{1}\right|} \int_{0}^{1}(1-q \tau)_{q}^{\alpha-2} \tau \nabla_{q} \tau\right] .
\end{aligned}
$$

Since

$$
\int_{0}^{t}(t-q \tau)_{\mathrm{q}}^{\alpha-1} \tau \nabla_{q} \tau=\int_{0}^{1} t^{\alpha+1}(1-q \tau)_{q}^{\alpha-1} \tau \nabla_{q} \tau
$$

we have

$$
\begin{aligned}
& |T x(t)-T y(t)| \\
& \leq d(r-1) M^{r-2}\|x-y\| \\
& \quad \times\left[\frac{1}{\Gamma_{q}(\alpha)} \int_{0}^{1} t^{\alpha+1}(1-q \tau)_{q}^{\alpha-1} \tau \nabla_{q} \tau\right. \\
& \quad+\frac{\left|a_{0}\right|}{\Gamma_{q}(\alpha)\left|1-a_{0}\right|} \int_{0}^{1}(1-q \tau)_{q}^{\alpha-1} \tau \nabla_{q} \tau \\
& \left.\quad+\frac{\left|a_{1}\right|\left(\left|a_{0}\right|+t\left|1-a_{0}\right|\right)}{\Gamma_{q}(\alpha-1)\left|1-a_{0}\right|\left|1-a_{1}\right|} \int_{0}^{1}(1-q \tau)_{q}^{\alpha-2} \tau \nabla_{q} \tau\right] .
\end{aligned}
$$

Using $q$-Beta function and the fact that $t \in[0,1]$, we get

$$
\begin{aligned}
& |T x(t)-T y(t)| \\
& \leq d(r-1) M^{r-2}\|x-y\| \\
& \times\left[\frac{1}{\Gamma_{q}(\alpha)} B_{q}(2, \alpha)+\frac{\left|a_{0}\right|}{\Gamma_{q}(\alpha)\left|1-a_{0}\right|} B_{q}(2, \alpha)\right. \\
& \left.\quad+\frac{\left|a_{1}\right|\left(\left|a_{0}\right|+\left|1-a_{0}\right|\right)}{\Gamma_{q}(\alpha-1)\left|1-a_{0}\right|\left|1-a_{1}\right|} \frac{[\alpha+1]_{q}}{[\alpha-1]_{q}} B_{q}(2, \alpha)\right] \\
& \leq d(r-1) M^{r-2} B_{q}(2, \alpha)\|x-y\| \\
& \times\left[\frac{1}{\Gamma_{q}(\alpha)}+\frac{\left|a_{0}\right|}{\Gamma_{q}(\alpha)\left|1-a_{0}\right|}\right. \\
& \left.\quad+\frac{\left|a_{1}\right|\left(\left|a_{0}\right|+\left|1-a_{0}\right|\right)[\alpha+1]_{q}}{\Gamma_{q}(\alpha)\left|1-a_{0}\right|\left|1-a_{1}\right|}\right]
\end{aligned}
$$

$$
\begin{aligned}
\leq d & (r-1) M^{r-2} B_{q}(2, \alpha)\|x-y\| \\
\times & {\left[\left(\left|1-a_{0}\right|\left|1-a_{1}\right|+\left|a_{0}\right|\left|1-a_{1}\right|\right.\right.} \\
& \left.+\left|a_{1}\right|\left(\left|a_{0}\right|+\left|1-a_{0}\right|\right)[\alpha+1]_{q}\right) \\
& \left.\times\left(\Gamma_{q}(\alpha)\left|1-a_{0}\right|\left|1-a_{1}\right|\right)^{-1}\right] \\
\leq & \frac{d(r-1) M^{r-2}}{\Gamma_{q}(\alpha+2)} \\
\times & {\left[\frac{\left(\left|1-a_{0}\right|+\left|a_{0}\right|\right)\left(\left|1-a_{1}\right|+\left|a_{1}\right|[\alpha+1]_{q}\right)}{\left|1-a_{0}\right|\left|1-a_{1}\right|}\right] } \\
\times & \|x-y\| \leq K\|x-y\|,
\end{aligned}
$$

where

$$
\begin{aligned}
K= & \frac{d(r-1) M^{r-2}}{\Gamma_{q}(\alpha+2)} \\
& \times\left[\frac{\left(\left|1-a_{0}\right|+\left|a_{0}\right|\right)\left(\left|1-a_{1}\right|+\left|a_{1}\right|[\alpha+1]_{q}\right)}{\left|1-a_{0}\right|\left|1-a_{1}\right|}\right] .
\end{aligned}
$$

By condition (43), we get $K<1$ which implies that $T$ is a contraction; therefore boundary value problem given in (19) has a unique solution.

Next, we give some examples to illustrate our results.

Example 8. Consider the following Boundary value problem

$$
\begin{gathered}
\nabla_{q}\left(\varphi_{7 / 3}\left({ }_{q} C_{0}^{3 / 2} x(t)\right)\right) \\
=4 t^{2}\left(2+\cos \left(\frac{\sqrt{\pi} x}{24}+\omega\right)\right), \quad t \in(0,1), \\
\nabla_{q}^{k} x(0)=0, \quad \text { for } k=2,3, \ldots, n-1, \\
x(0)=\frac{1}{2} x(1), \\
\nabla_{q} x(0)=\frac{1}{2} \nabla_{q} x(1),
\end{gathered}
$$

where

$$
\begin{gathered}
p=\frac{7}{3}, \quad \alpha=\frac{3}{2}, \quad \delta=4, \\
a_{0}=\frac{1}{2}, \quad a_{1}=\frac{1}{2} .
\end{gathered}
$$

Then $r=7 / 4$, and take $\delta=4, \lambda=1$, and $d=\sqrt{\pi} / 6$. Using Theorem 1 and the fact that $[3 / 2]_{q}>1$ for any fixed $q \in(0,1)$, 
we have

$$
\begin{aligned}
& \lambda^{2-r} \frac{\Gamma_{q}(\delta(r-2)+2+\alpha)}{(r-1) \Gamma_{q}(\delta(r-2)+2)} \\
& \times\left[\left(\left|1-a_{0}\right|\left|1-a_{1}\right|\right)\right. \\
& \times\left(\left(\left|1-a_{0}\right|+\left|a_{0}\right|\right)\right. \\
& \left.\left.\times\left(\left|1-a_{1}\right|+\left|a_{1}\right|[\delta(r-2)+\alpha+1]_{q}\right)\right)^{-1}\right] \\
& =\frac{2}{3}\left[\frac{[3 / 2]_{q} \Gamma_{q}(3 / 2)}{\left(1+[3 / 2]_{q}\right)}\right]>\frac{2}{3}\left[\frac{[3 / 2]_{q} \Gamma_{q}(3 / 2)}{\left([3 / 2]_{q}+[3 / 2]_{q}\right)}\right] \\
& >\frac{1}{3} \Gamma\left(\frac{3}{2}\right)=\frac{\sqrt{\pi}}{6}=d>0, \\
& K=d(r-1) \lambda^{r-2} \frac{\Gamma_{q}(\delta(r-2)+2)}{\Gamma_{q}(\delta(r-2)+2+\alpha)} \\
& \times\left[\frac{\left(\left|1-a_{0}\right|+\left|a_{0}\right|\right)\left(\left|1-a_{1}\right|+\left|a_{1}\right|[\delta(r-2)+\alpha+1]_{q}\right)}{\left|1-a_{0}\right|\left|1-a_{1}\right|}\right] \\
& =\frac{\sqrt{\pi}}{4 \Gamma_{q}(5 / 2)}\left[\left(1+\left[\frac{3}{2}\right]_{q}\right)\right] \\
& <\frac{\sqrt{\pi}}{4[3 / 2]_{q} \Gamma_{q}(3 / 2)}\left[\left(\left[\frac{3}{2}\right]_{q}+\left[\frac{3}{2}\right]_{q}\right)\right] \leq \frac{\sqrt{\pi}}{2 \Gamma(3 / 2)}=1 .
\end{aligned}
$$

Moreover, it can be easily seen that

$$
\begin{aligned}
{[\delta]_{q} \lambda t^{\delta-1} } & =[4]_{q} t^{3} \leq 4 t^{2}\left(2+\cos \left(\frac{\sqrt{\pi} x}{24}+\omega\right)\right) \\
& =f(t, x) .
\end{aligned}
$$

Finally,

$$
\begin{aligned}
|f(t, x)-f(t, y)| \\
=\mid 4 t^{2}\left(2+\cos \left(\frac{\sqrt{\pi} x}{24}+\omega\right)\right) \\
\quad-4 t^{2}\left(2+\cos \left(\frac{\sqrt{\pi} y}{24}+\omega\right)\right) \mid \\
=4 t^{2}\left|\cos \left(\frac{\sqrt{\pi} x}{24}+\omega\right)-\cos \left(\frac{\sqrt{\pi} y}{24}+\omega\right)\right| \\
\leq 4\left|\left(\frac{\sqrt{\pi} x}{24}+\omega\right)-\left(\frac{\sqrt{\pi} y}{24}+\omega\right)\right| \\
=\frac{\sqrt{\pi}}{6}|x-y| .
\end{aligned}
$$

Therefore as a consequence of Theorem 4, boundary value problem given in (53) has a unique solution.
Example 9. Consider the following boundary value problem:

$$
\begin{gathered}
\nabla_{q}\left(\varphi_{9 / 4}\left(\varphi_{31 / 15}\left({ }_{q} C_{0}^{3 / 2} x(t)\right)\right)\right) \\
=4 t^{2}\left(2+\cos \left(\frac{\sqrt{\pi} x}{24}+\omega\right)\right), \quad t \in(0,1), \\
\nabla_{q}^{k} x(0)=0, \quad \text { for } k=2,3, \ldots, n-1, \\
x(0)=\frac{1}{2} x(1), \\
\nabla_{q} x(0)=\frac{1}{2} \nabla_{q} x(1) .
\end{gathered}
$$

Then

$$
\begin{aligned}
\varphi_{9 / 4}\left(\varphi_{31 / 15}(s)\right) & =\varphi_{9 / 4}\left(|s|^{1 / 15} s\right)=\left.\left.|| s\right|^{1 / 15} s\right|^{(9 / 4)-2}|s|^{1 / 15} s \\
& =|s|^{(1 / 15)(1 / 4)}|s|^{1 / 4}|s|^{1 / 15} s \\
& =|s|^{(1 / 60)+(1 / 4)+(1 / 15)} s=|s|^{1 / 3} s \\
& =|s|^{(7 / 3)-2} s=\varphi_{7 / 3}(s) .
\end{aligned}
$$

Therefore boundary value problem given in (58) reduces to the boundary value problem given in (53), and it has a unique solution.

Example 10. Now consider the following antiperiodic boundary value problem:

$$
\begin{gathered}
\nabla_{q}\left(\varphi_{7 / 4}\left({ }_{q} C_{0}^{3 / 2} x(t)\right)\right) \\
=\left(\sin ^{2}\left(\frac{\sqrt{\pi} x}{40}+\omega\right)\right), \quad t \in(0,1), \\
\nabla_{q}^{k} x(0)=0, \quad \text { for } k=2,3, \ldots, n-1, \\
x(0)=-x(1), \\
\nabla_{q} x(0)=-\nabla_{q} x(1),
\end{gathered}
$$

where

$$
p=\frac{7}{4}, \quad \alpha=\frac{3}{2}, \quad a_{0}=-1, \quad a_{1}=-1 \text {. }
$$


Then $r=7 / 3$, and take $d=\sqrt{\pi} / 20$. Using Theorem 1 and taking $g(t)=1$, we get that

$$
M=1,
$$

$$
\begin{aligned}
& \frac{\Gamma_{q}(\alpha+2)}{(r-1) M^{r-2}} \\
& \quad \times\left[\frac{\left|1-a_{0}\right|\left|1-a_{1}\right|}{\left(\left|1-a_{0}\right|+\left|a_{0}\right|\right)\left(\left|1-a_{1}\right|+\left|a_{1}\right|[\alpha+1]_{q}\right)}\right] \\
& =\left[\frac{\Gamma_{q}(7 / 2)}{\left(2+[5 / 2]_{q}\right)}\right]>\left[\frac{[5 / 2]_{q}[3 / 2]_{q} \Gamma_{q}(3 / 2)}{\left(2[5 / 2]_{q}+[5 / 2]_{q}\right)}\right] \\
& =\frac{[3 / 2]_{q} \Gamma_{q}(3 / 2)}{3}>\frac{\Gamma(3 / 2)}{3}=\frac{\sqrt{\pi}}{6}>\frac{\sqrt{\pi}}{20}=d .
\end{aligned}
$$

On the other hand,

$$
\begin{aligned}
&|f(t, x)-f(t, y)| \leq\left|\sin ^{2}\left(\frac{\sqrt{\pi} x}{40}+\omega\right)-\sin ^{2}\left(\frac{\sqrt{\pi} y}{40}+\omega\right)\right| \\
& \leq \frac{\sqrt{\pi}}{20}|x-y|, \quad \text { for } t \in[0,1], x, y \in \mathbb{R}, \\
& K=\frac{d(r-1) M^{r-2}}{\Gamma_{q}(\alpha+2)} \\
&\left.=\frac{\sqrt{\left(\left|1-a_{0}\right|+\left|a_{0}\right|\right)\left(\left|1-a_{1}\right|+\left|a_{1}\right|[\alpha+1]_{q}\right)}}{20 \Gamma_{q}(7 / 2)\left[2+\left[\frac{5}{2}\right]_{q}\right]}\right] \\
&<\frac{\sqrt{\pi}\left[1-a_{1}|| 1-a_{0}\left[\frac{5}{2}\right]_{q}\right]}{20[5 / 2]_{q}[3 / 2]_{q} \Gamma_{q}(3 / 2)}[3 \sqrt{\pi} \\
&= \frac{3 \sqrt{\pi}}{20[3 / 2]_{q} \Gamma_{q}(3 / 2)}<\frac{3}{20 \Gamma(3 / 2)}=\frac{3}{10}<1 .
\end{aligned}
$$

Therefore by Theorem 7 , the antiperiodic boundary value problem given in (60) has a unique solution.

\section{References}

[1] T. Abdeljawad, B. Benli, and D. Baleanu, "A generalized $q$-Mittag-Leffler function by $q$-Captuo fractional linear equations," Abstract and Applied Analysis, vol. 2012, Article ID 546062, 11 pages, 2012.

[2] T. Abdeljawad and D. Baleanu, "Fractional differences and integration by parts," Journal of Computational Analysis and Applications, vol. 13, no. 3, pp. 574-582, 2011.

[3] T. Abdeljawad and D. Baleanu, "Caputo $q$-fractional initial value problems and a $q$-analogue Mittag-Leffler function," Communications in Nonlinear Science and Numerical Simulation, vol. 16, no. 12, pp. 4682-4688, 2011.

[4] R. P. Agarwal, "Certain fractional $q$-integrals and $q$-derivatives," vol. 66, pp. 365-370, 1969.
[5] W. A. Al-Salam, "Some fractional q-integrals and q-derivatives," Proceedings of the Edinburgh Mathematical Society. Series II, vol. 15, pp. 135-140, 1966.

[6] T. Chen and W. Liu, "An anti-periodic boundary value problem for the fractional differential equation with a $p$-Laplacian operator," Applied Mathematics Letters, vol. 25, no. 11, pp. 1671$1675,2012$.

[7] T. Ernst, "The history of $q$-calculus and new methods. (Licentiate Thesis)," U.U.D.M. Report, 2000.

[8] F. Jarad, T. Abdeljawad, and D. Baleanu, "Stability of $q$-fractional non-autonomous systems," Nonlinear Analysis. Real World Applications. An International Multidisciplinary Journal, vol. 14, no. 1, pp. 780-784, 2013.

[9] I. Podlubny, Fractional Differential Equations, Academic Press, New York, NY, USA, 1999.

[10] S. G. Samko, A. A. Kilbas, and O. I. Marichev, Fractional Integrals and Derivatives, Gordon and Breach Science, Yverdon, Switzerland, 1993.

[11] G. E. Andrews, R. Askey, and R. Roy, Special Functions, Cambridge University Press, Cambridge, UK, 1999.

[12] F. H. Jackson, "A generalization of the functions $\Gamma(n)$ and $x^{n}$," Proceedings of the Royal Society of London, no. 74, pp. 64-72, 1904.

[13] J. Thomae, "Beitrage zur Theorie der durch die Heinesche Reihe," Journal für die Reine und Angewandte Mathematik, no. 70, pp. 258-281, 1869.

[14] X. Liu, M. Jia, and X. Xiang, "On the solvability of a fractional differential equation model involving the $p$-Laplacian operator," Computers \& Mathematics with Applications. An International Journal, vol. 64, no. 10, pp. 3267-3275, 2012. 




Advances in

Operations Research

mansans



The Scientific World Journal
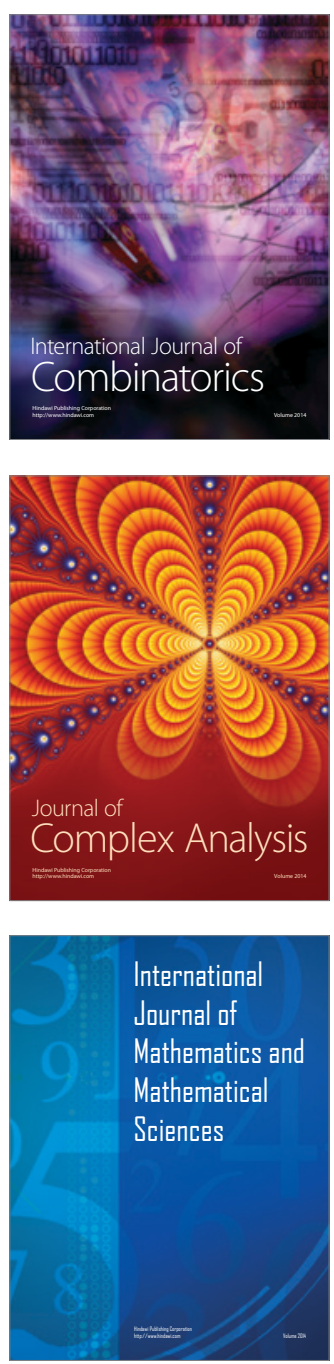
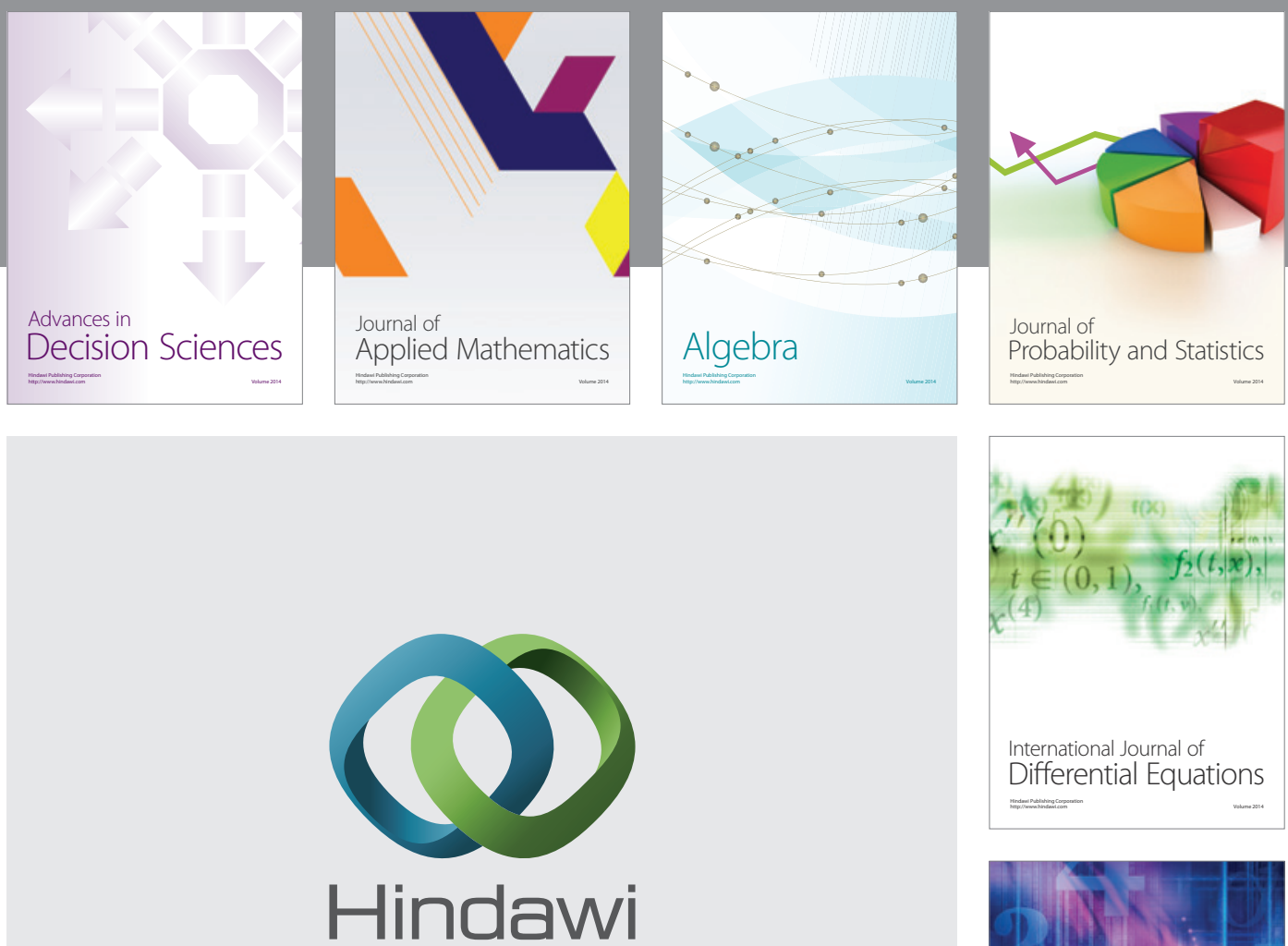

Submit your manuscripts at http://www.hindawi.com
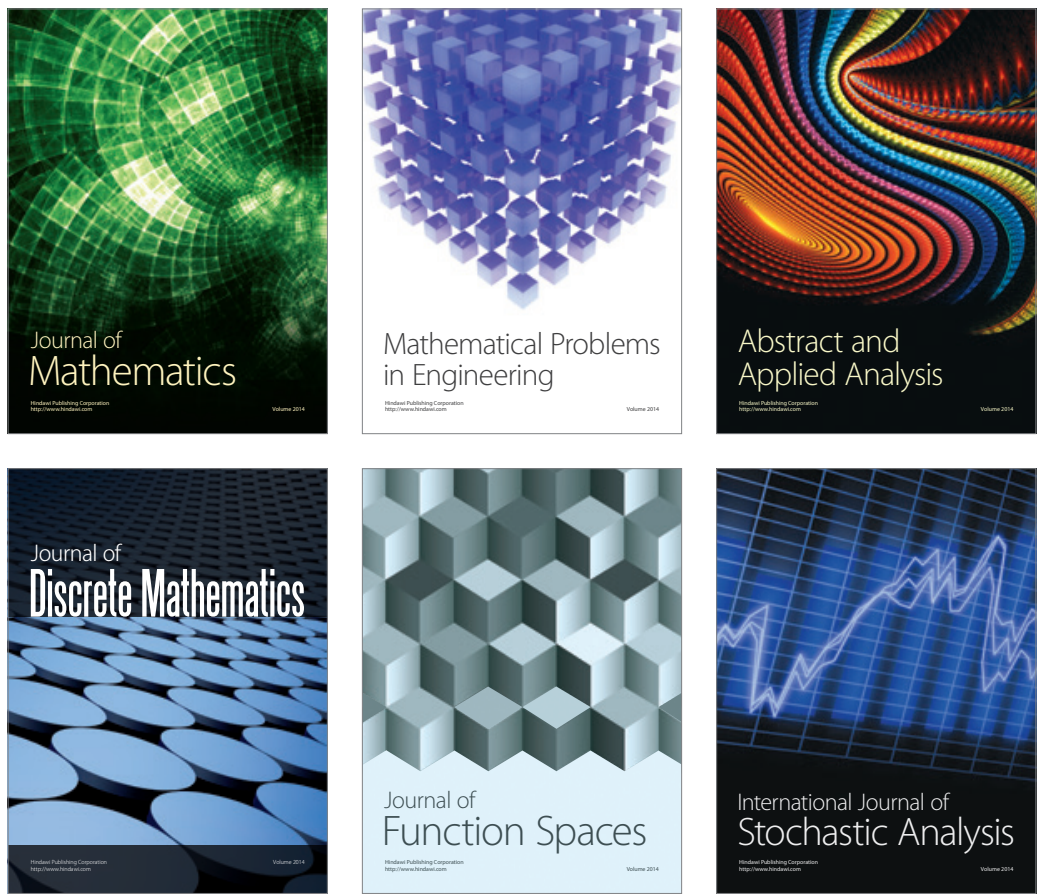

Journal of

Function Spaces



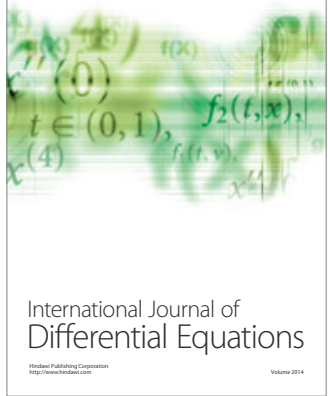
